\title{
Determinação experimental da constante de Planck pela observação da corrente de descarga de um capacitor ${ }^{+*}$
}

Erick Santana dos Santos ${ }^{1}$

Instituto Federal da Bahia

Roberto dos Santos Menezes $\mathrm{Jr}^{2}$

Instituto Federal da Bahia

Victor Mancir da Silva Santana ${ }^{3}$

Instituto de Física - UFBA

Salvador - BA

\section{Resumo}

Neste artigo nós apresentamos um método experimental para a determinação da constante de Planck, baseado na medição da corrente de descarga de um capacitor sobre um LED, sem o uso de resistores. Para tanto, utilizamos um capacitor ligado em paralelo a um LED e observamos a tensão no capacitor durante sua descarga juntamente com a corrente que passa através do amperímetro, ligado em série ao LED. No instante em que a corrente torna-se zero, a tensão aplicada sobre o LED corresponde à tensão de corte, pela qual podemos determinar a constante de Planck. Além disso, nós discutimos alguns conceitos importantes a respeito dos aspectos quânticos do funcionamento do LED e apresentamos os resultados obtidos a partir desta metodologia, com algumas discussões.

Palavras-chave: Constante de Planck; LED; Capacitor.

\footnotetext{
${ }^{+}$Experimental determination of the Planck's constant by observation of the discharge current of a capacitor

* Recebido: dezembro de 2014.

Aceito: junho de 2015.

1 E-mail: erick_santana@ifba.edu.br

2 E-mail: rsmjr@ifba.edu.br

3 E-mail: santana.victor86@gmail.com
} 


\begin{abstract}
In this paper, we present an experimental method for the determination of Planck's constant, based on the measurement of the discharge current of a capacitor on a LED, without the use of resistors. To develop this, we use a capacitor connected in parallel to a LED and observe the voltage on the capacitor during its discharge along with the current flowing through the ammeter connected in series to the LED. At the instant when the current becomes zero, the voltage applied on the LED corresponds to the cutoff voltage, by which we can determine the Planck's constant. Moreover, we treat some important concepts about the quantum phenomena of the LED operation and present the results from this methodology, with some discussions.
\end{abstract}

Keywords: Planck's Constant; LED; Capacitor.

\title{
I. Introdução
}

A atividade experimental é uma importante parte que integra o ensino de física (FORÇA et al., 2011) e essa importância tem sido, já há algum tempo, amplamente discutida por diversos pesquisadores. Uma consequência dessas discussões é a grande quantidade de propostas dessas atividades surgidas nos últimos anos, nos diversos níveis de ensino (MOREIRA; PENIDO, 2009; AZEVEDO et al., 2009), porém essa tendência ainda não é devidamente acompanhada pelas escolas. Especialmente no que tange à física moderna, grande parte da atividade experimental depende de aparatos complexos e caros, ficando, portanto, sua aquisição, fora da realidade da grande maioria das escolas, ou mesmo das faculdades e universidades. Assim, a proposta de atividades experimentais, principalmente no campo da física moderna, que utilizem materiais de fácil aquisição configura-se como uma alternativa a essas dificuldades. Nesse sentido, pretendemos apresentar um experimento de simples realização, usando materiais de fácil aquisição, que se enquadra no contexto da física moderna.

O nosso experimento visa a determinação da constante de Planck por meio da medição da tensão de corte de lâmpadas do tipo LED. A metodologia aqui empregada, no entanto, difere daquelas encontradas em outros experimentos que utilizam um potenciômetro na configuração de divisor de tensão para regular a diferença de potencial sobre os LEDs (ver figura 1), e cuja medida é feita variando-se manualmente esse potenciômetro (CAVALCANTE; HAAG, 2005; HORIGUTI; OLIVEIRA, 2008; INDELICATO et al., 2013; MOREHOUSE, 1998; MOURA, 2011). Nesses experimentos, a tensão sobre o LED é variada desde um ponto em que não há a emissão de luz (e, portanto, não há passagem de corrente elétrica pelo LED) a outro, um pouco além da emissão. Constrói-se, então, um gráfico com os valores de corrente obtidos em função da tensão. Em seguida, a partir da reta obtida dos valores das tensões e correntes pós-emissão de luz, determina-se a tensão de corte por extrapolação e, desse valor, obtém-se a constante de 
Planck. Nessa configuração, além dos erros intrínsecos associados aos instrumentos de medida, a precisão do resultado depende do tipo de potenciômetro escolhido, pois, se sua sensibilidade não for adequada, os valores de tensão medidos sobre o LED terão intervalos de medição relativamente altos, o que impossibilita uma boa determinação do valor da tensão de corte. Além disso, assume-se que o comportamento desse gráfico, um pouco após o limiar da emissão (início da passagem de corrente pelo LED), segue o mesmo comportamento linear dos demais intervalos, o que não condiz com os resultados experimentais (HORIGUTI; OLIVEIRA, 2008; MOURA, 2011, RAYCHAUDHURI, 2011), como pode ser observado na Fig. 2.

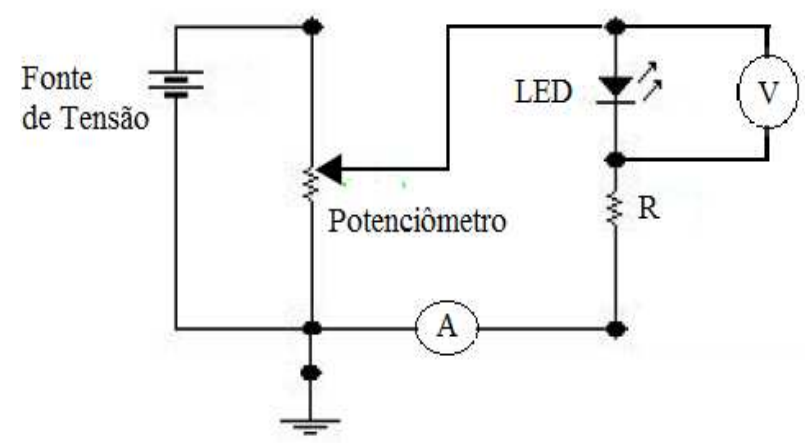

Fig. 1 - Exemplo de circuito geralmente utilizado na determinação da constante de Planck.

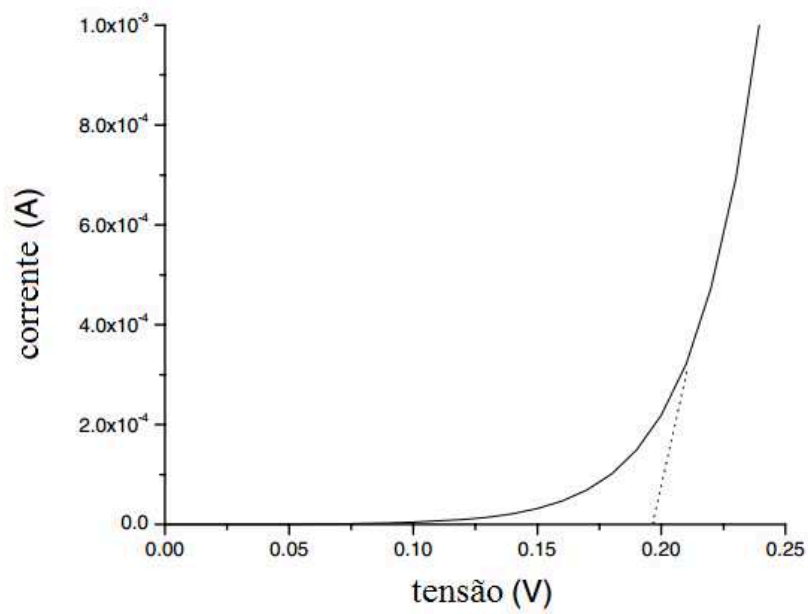

Fig. 2 - Gráfico corrente x tensão obtido para um LED. Observa-se nele um comportamento não linear para valores de corrente próximos de zero (Fonte: RAYCHAUDHURI, 2011).

Em nossa metodologia, diferentemente da empregada nas referências anteriormente citadas, a medida é feita diretamente, sem o uso de extrapolação gráfica. Dessa forma, os erros associados às medidas dependem somente da precisão dos instrumentos utilizados (neste caso, o voltímetro e amperímetro) e da qualidade dos LEDs. Além disso, pode-se também 
utilizar LEDs que emitam fora da região visível do espectro eletromagnético (LEDs infravermelhos e ultravioletas) e até mesmo diferentes tipos de diodos. Outra vantagem reside na simplicidade da medida, visto que outros autores (THURMAN, 2013; ZHOU; CLONINGER, 2008) utilizaram uma metodologia semelhante à apresentada neste artigo, porém com sistemas de aquisição de dados mais complexos, em que as tensões fornecidas ao LED e a corrente medida são controladas por um software em um computador. Além disso, eles utilizam resistências adicionais ao circuito, o que leva à necessidade de conhecimentos e interpretações mais detalhadas de diferentes fenômenos físicos no tratamento experimental (circuito RC em série com um LED, por exemplo) que estão fora do escopo da abordagem experimental. Na montagem aqui sugerida, o relativo baixo custo e a facilidade na aquisição dos materiais necessários para sua aplicação auxiliam na compreensão do experimento e na sua execução.

Na próxima seção, discutiremos os aspectos físicos relacionados ao experimento; na seção III faremos uma descrição do procedimento experimental e materiais necessários à sua execução; na seção IV apresentaremos os resultados obtidos e na seção V faremos algumas considerações finais.

\section{A Física Quântica e o LED}

A constante $h$ foi introduzida pela primeira vez por Max Planck, no ano de 1901, para explicar a distribuição da intensidade espectral da radiação de um corpo negro (e que lhe rendeu, em 1918, o prêmio Nobel de Física). Os corpos negros podem ser definidos como corpos cuja superfície absorve toda a faixa do espectro da radiação incidente sobre eles e que, quando aquecidos, emitem a máxima intensidade de radiação possível para uma dada temperatura. Nesta época, conhecia-se como a energia da radiação térmica de um corpo, mantido a uma determinada temperatura, se distribui entre as diversas frequências, porém nenhuma teoria havia sido capaz de propor uma explicação destes resultados experimentais, apesar das tentativas de Wien e de Rayleigh e Jeans (EISBERG; RESNICK, 1994; HEWITT, 2011). Para resolver esse problema, Planck teve que assumir que a energia da radiação aprisionada na cavidade do corpo negro, para uma dada frequência, não se distribuía continuamente, como era previsto pela física clássica, mas de maneira discreta, com valores proporcionais à essa mesma frequência. Dessa hipótese surgiu a famosa relação da quantização da energia

$$
E=h v \text {, }
$$

na qual $h$ ficou conhecida como constante de Planck, cujo valor é $6,626 \times 10^{-34} \mathrm{~J} \cdot \mathrm{s}$ (MOHR et $a l ., 2012)$. Com isso, Planck pôde então estabelecer a relação entre a intensidade da radiação emitida por um corpo negro, $I(\lambda)$, e o comprimento de onda, $\lambda$, para uma determinada temperatura $T$,

$$
I(\lambda)=\frac{2 \pi c^{2}}{\lambda^{5}} \frac{h}{e^{h c / \lambda k T-1}}
$$

na qual $k=1,38 \times 10^{-23} \mathrm{~J} / K$ é a constante de Boltzmann. 
Alguns anos depois, em 1905, Albert Einstein utilizou a equação (1) para explicar alguns aspectos do efeito fotoelétrico (que consiste na emissão de elétrons por um condutor quando exposto a radiações de altas frequências, primeiramente observado por A. E. Becquerel, em 1839, e confirmado por Heinrich Hertz, em 1887) que também não podiam ser explicados pela física clássica (EISBERG; RESNICK, 1994).

Os LEDs (do inglês, light emitting diode ou "diodo emissor de luz") são dispositivos semicondutores que emitem radiação eletromagnética (NUSSENZVEIG, 2009), desde a região ultravioleta até o infravermelho próximo, quando uma tensão é aplicada sobre os seus terminais. Devido à tecnologia utilizada no processo de fabricação, os LEDs emitem radiação em uma faixa espectral (intervalo de comprimentos de onda) cujo pico determina a frequência predominante na emissão (comprimento de onda de máxima emissão), definindo a cor do LED, e cuja largura pode conter algumas dezenas de nanômetros (CAVALCANTE; HAAG, 2005; KRAFTMAKHER, 2011; RAYCHAUDHURI, 2011). Devido à largura relativamente estreita em torno do pico de emissão, podemos considerar que a radiação emitida é aproximadamente monocromática (ou seja, em um único comprimento de onda). Ver Fig. 3.

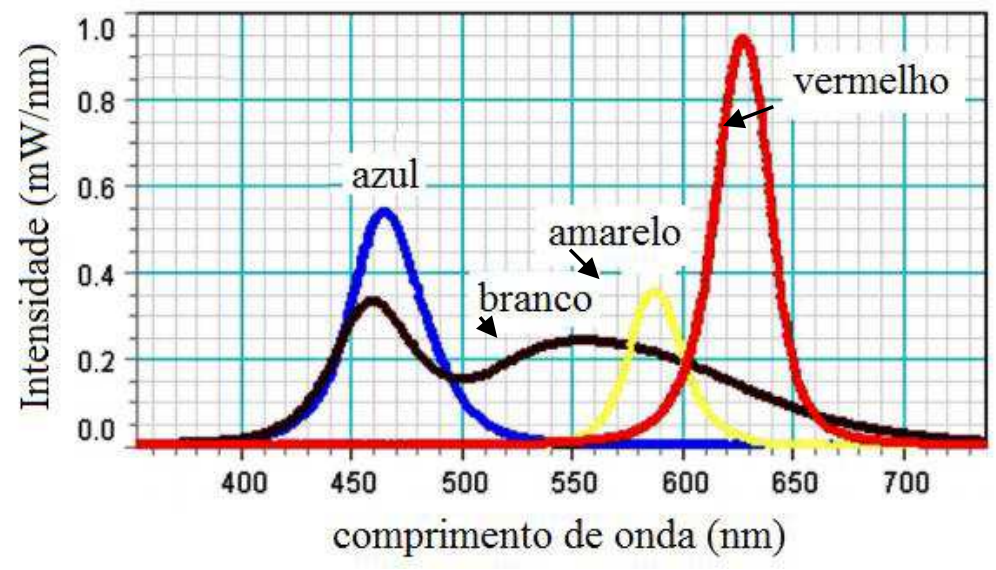

Fig. 3 - Exemplo de espectros de emissão de diversos LEDs. (Fonte: KRAFTMAKHER, 2011)

Os LEDs são constituídos por uma junção de semicondutores do tipo $n$ e do tipo $p$. Um semicondutor é do tipo $n$ quando os portadores majoritários de corrente elétrica são elétrons da banda de condução. As bandas de energia (banda de condução e valência, importantes na descrição da junção entre semicondutores do tipo $n$ e $p$ ) são formadas quando ocorrem desdobramentos dos níveis de energia discretos dos átomos isolados durante a formação do cristal. A banda de condução é o nível energético mais distante do núcleo, ou seja, desdobramento da última camada discreta, e a banda de condução é o desdobramento do nível discreto anterior. A diferença entre esses níveis de energia é comumente chamado de gap (do inglês, "lacuna") ou barreira de potencial.

A construção do semicondutor tipo $n$ pode ser feita por meio do processo de "dopagem", que consiste na adição de átomos de um material diferente (impureza) ao semicondutor 
da matriz original (por exemplo, adicionando um átomo de fósforo, que pode realizar até cinco ligações químicas (pentavalente), ao silício, que pode realizar até quatro ligações (tetravalente)). O semicondutor passa, então, a ter elétrons em excesso e fracamente ligados ao núcleo original, de tal forma que pode doá-los, podendo assim participar da corrente elétrica.

Analogamente, dizemos que um semicondutor é do tipo $p$ quando adicionamos átomos ao semicondutor de tal forma que esse possa capturar um elétron já existente no material, deixando um nível de energia vazio que pode ser ocupado por outro elétron (por exemplo, adicionando gálio (trivalente), ao silício (tetravaente)). Nesse caso, a matriz resultante terá "buracos" ou "lacunas", que são níveis de energia pertencentes à banda de valência, desocupados pelos elétrons do semicondutor (REZENDE, 2004, ALVES; SILVA, 2008). A Fig. 4 ilustra um semicondutor de silício (Si) e suas dopagens tipo $n$ e tipo $p$.

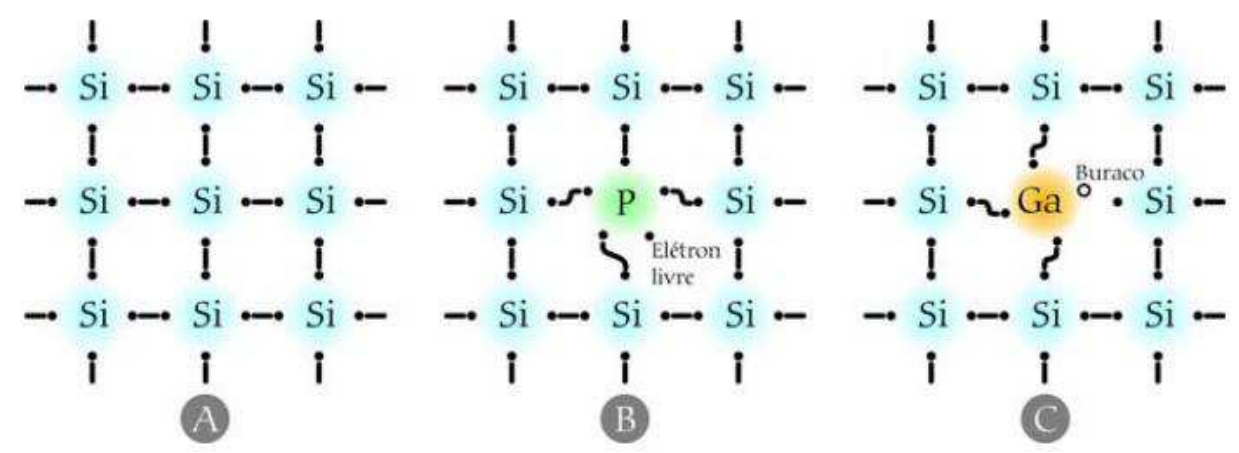

Fig. 4 - (A) Semicondutor formado por silício (Si). (B) A introdução de átomos de fósforo $(P)$ ao semicondutor adiciona um elétron que pode mover-se livremente no material semicondutor tipo $n$. (C) A introdução de gálio $(G a)$ ao semicondutor gera um nível de energia desocupado (buraco), que pode capturar elétrons do semicondutor, deixando novos buracos nele - semicondutor tipo p (Fonte: ALVES; SILVA, 2008).

Quando a junção pn é formada, para que haja a passagem de corrente, é necessário que se forneça uma energia, $E_{g}$, suficiente para que os elétrons do semicondutor tipo $n$ vençam a barreira de potencial e, com isso, passem para a banda de condução, deixando um buraco na banda de valência (forma-se um par elétron-buraco). Isso pode ser conseguido submetendo o diodo a uma diferença de potencial $V_{c}=E_{g} / e$. Uma vez na banda de condução, esses elétrons podem migrar para o semicondutor tipo $p$ onde se recombinam com os buracos desse semicondutor, emitindo a diferença de energia em forma de radiação (COLINGE; COLINGE, 2000). Ver Fig. 5. A energia $E_{g}$ necessária para superar a barreira de potencial é bem definida para cada LED, o que ocasiona a emissão de radiação em um comprimento de onda bem característico.

Pelo fato dessa queda de energia se dar apenas na transição $n-p$, e esta lacuna de energia ter um valor bem definido, a radiação emitida é aproximadamente monocromática e obedece a relação de Einstein para a energia de um fóton apresentada na equação (1) (HALLIDAY et. 
al., 2004). Dessa forma, um quanta de energia $h v$ é necessário para superar a barreira de potencial e criar um par elétron-buraco, o que acarretará na posterior emissão de um fóton de mesma energia (ver Fig. 5). Assim, temos que
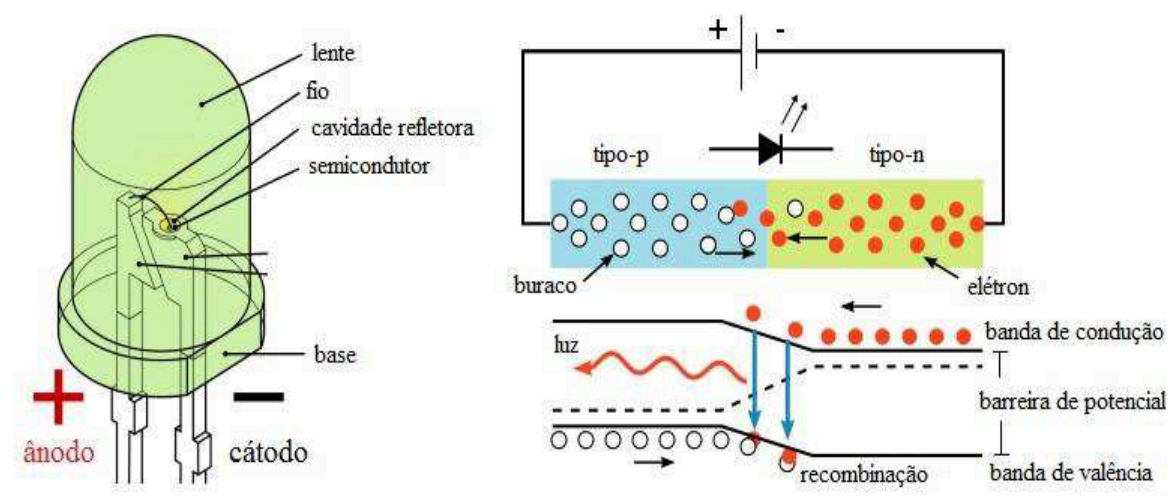

Fig. 5 - Esquemas representando um LED e seu processo de emissão de luz (Fonte: MOURA, 2011).

$$
E_{g}=h v=\frac{h c}{\lambda}=e V_{c}
$$

sendo $c$ a velocidade da luz no vácuo $\left(3,0 \times 10^{8} \mathrm{~m} / \mathrm{s}\right), e$ a carga do elétron $\left(1,602 \times 10^{-19} \mathrm{C}\right)$ e $h$ a constante de Planck a ser determinada $\left(6,63 \times 10^{-34} \mathrm{~J} \cdot \mathrm{s}\right)$. Logo, conhecendo-se o comprimento de onda de máxima de emissão de alguns LEDs, $\lambda$, e medindo-se sua tensão mínima de emissão (ou de corte da corrente), $V_{c}$, podemos determinar o valor da constante $h$.

\section{Descrição experimental}

Para a execução do experimento é necessário a utilização dos seguintes materiais (ver Fig. 6):

- LEDs de diversas cores: laranja, amarelo, vermelho, verde, azul e infravermelho;

- Protoboard para modelagem eletrônica;

- Fios de conexão (podem ser fios de internet ou fios apropriados para prototipagem em Protoboard);

- Bateria de $3 \mathrm{~V}$ (de lítio, daquelas encontradas em produtos eletrônicos) ou duas pilhas AA em série;

- Capacitor de $1000 \mu \mathrm{F}$;

- Microamperímetro digital (fundo de escala $100 \mu \mathrm{A}$ ou menor);

- Voltímetro digital (fundo de escala $20 \mathrm{~V}$ ). 
Em nosso experimento, determinaremos o instante em que o LED deixa de emitir radiação através da leitura da corrente que atravessa o amperímetro a ele associado em série. A nulidade dessa corrente indicará o instante do término da emissão. Para tanto, devemos, primeiramente, montar o circuito como mostrado na Fig. 7.

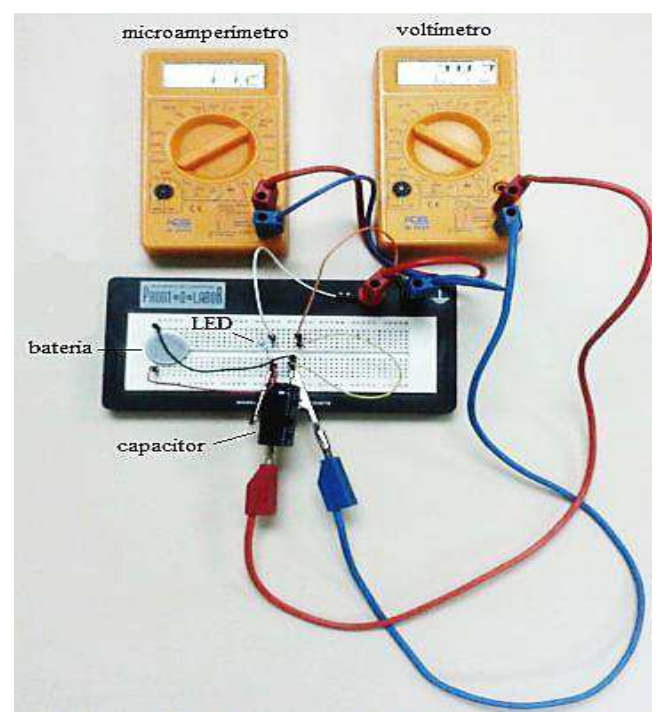

Fig. 6-Montagem realizada para medir a tensão mínima de emissão do LED.

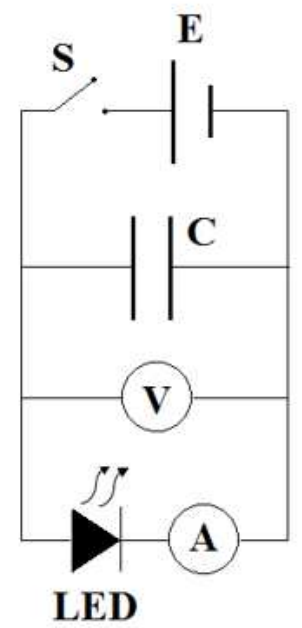

Fig. 7 - Esquema do circuito para determinação da constante de Planck.

O circuito da Fig. 7 apresenta a fonte de tensão $E$, o capacitor $C$ e o voltímetro $V$, ligados em paralelo, e o LED, associado em série a um microamperímetro. Ao ligarmos a chave $S$, o capacitor, após um pequeno intervalo de tempo, atinge entre seus terminais o valor máximo de tensão, ou seja, o valor da tensão da fonte $E(\approx 3 V)$. Neste instante a corrente no LED atinge o valor máximo para essa configuração e ele brilhará. Após liberada a chave, o capacitor começará a descarregar através do LED e do voltímetro, mas, como este último possui uma resistência muito grande comparada à do microamperímetro, podemos considerá-lo como ideal, de modo que iremos assumir que toda a corrente passará apenas pela associação do LED com o amperímetro. A corrente de descarga, e a consequente diminuição do brilho no LED, pode ser 
acompanhada instantaneamente pelo microamperímetro. O voltímetro nos dará a tensão nos terminais do LED nesse processo.

Quando o capacitor atingir a tensão de corte $V_{c}$, ele não será mais capaz de alimentar os elétrons com energia suficiente para vencerem a barreira de potencial e a corrente cessará (TAVOLARO; CAVALCANTE, 2003). Este instante pode ser determinado através da leitura nula no microamperímetro. Medindo-se esse valor de tensão para LEDs de diferentes comprimentos de onda (diferentes cores), é possível construir um gráfico $V_{c} \times 1 / \lambda$, cujo coeficiente angular, $\alpha$, será (ver equação (3))

$$
\alpha=\frac{h c}{e}
$$

Conhecidos, então, os valores das constantes $c$ e $e$ é possível a determinação do valor de $h$.

\section{Resultados}

Realizando o procedimento descrito na seção anterior, medimos a tensão de corte para LEDs de diferentes comprimentos de ondas e obtivemos os resultados ilustrados na tabela I, onde indicamos também os comprimentos de onda utilizados para cada um deles.

Tabela I: Valores de comprimentos de onda utilizados para cada LED e suas respectivas tensões de corte.

\begin{tabular}{l|c|c}
\hline LED (cor) & $\lambda(\mathrm{nm})$ & $V_{c}(V)$ \\
\hline Infravermelho & 850 & 1,32 \\
\hline Vermelho & 635 & 1,70 \\
\hline Laranja & 585 & 1,93 \\
\hline Verde & 525 & 2,47 \\
\hline Azul & 470 & 2,41 \\
\hline
\end{tabular}

$\mathrm{O}$ erro de leitura do voltímetro foi de $\pm 0,01 \mathrm{~V}$ e do microamperímetro de $\pm 0,1 \mu \mathrm{A}$. A tensão inicial em todas as leituras foi de $E=2,67 \mathrm{~V}$. Com os valores da tabela I, foi possível plotar o gráfico de $V_{c} \times 1 / \lambda$, apresentado na Fig. 8 .

Aplicando-se a regressão linear aos dados da tabela I, obtém-se

$$
V_{c}=\frac{1,29 \times 10^{-6}}{\lambda}-0,23,
$$

com uma concordância de $R^{2}=0,96$ e coeficiente angular, $\alpha=(1,29 \pm 0,13) \times 10^{-6} \mathrm{~J} \cdot \mathrm{m} / \mathrm{C}$. Este último resultado nos permite calcular a constante de Planck, por meio da equação (4), obtendo

$$
h=(6,89 \pm 0,69) \times 10^{-34} J \cdot s,
$$




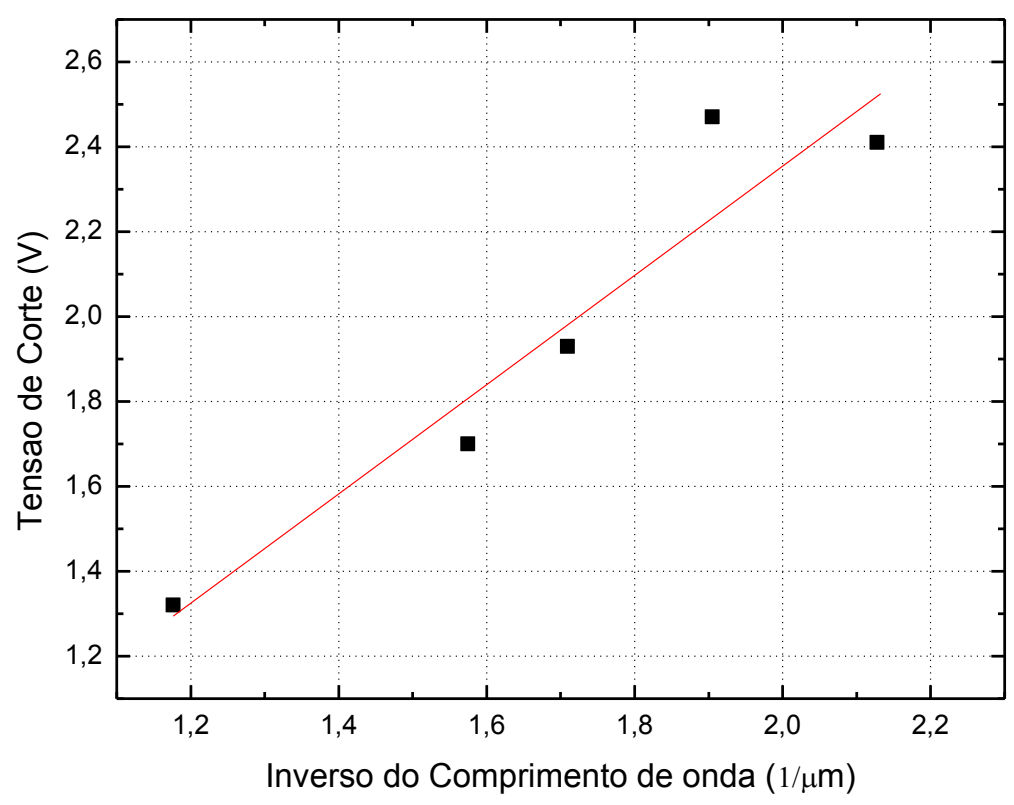

Fig. 8 - Ajuste linear dos pontos obtidos no experimento.

que difere do valor encontrado na literatura, $h=6,63 \times 10^{-34} \mathrm{~J} \cdot s$ (MOHR et. al., 2012), em apenas 3,9\%. Esse erro se deve, em grande parte, ao valor do comprimento de onda utilizado para os LEDs, uma vez que os obtivemos de tabelas encontradas na rede, de acordo com as características físicas dos mesmos, mas esses valores podem variar significativamente de acordo com o fabricante. Além disso, devemos notar que consideramos os LEDs como fontes monocromáticas, mas, como já dissemos, a sua faixa espectral pode ter uma largura de algumas dezenas de nanômetros. Outro fator que deve ser levado em conta é o da precisão dos instrumentos de medida, uma vez que o instante em que se observa a leitura nula da corrente (e anotase a tensão de corte) dependerá da precisão do microamperímetro.

Para a aplicação dessa atividade experimental, devido à grande divergência que existe entre comprimentos de onda de LEDs de uma mesma cor, sugerimos a realização prévia do experimento por parte do professor, ou seja, a medida das tensões de corte para os diferentes LEDs disponíveis, e a adequação dos comprimentos de onda de acordo com tabelas encontradas na rede (como, por exemplo, a encontrada no site <http://baudaeletronica.blogspot.com.br/2008/03/tabela-de-leds.html>). Conhecidos esses comprimentos de onda, pode-se selecionar aqueles que proporcionem um melhor ajuste do coeficiente angular da regressão linear (ou seja, para valores próximos a $1,24 \times 10^{-6} \mathrm{~J} \cdot \mathrm{m} / \mathrm{C}$ ), fornecendo assim um valor mais preciso para constante de Planck.

Outra alternativa, para laboratórios mais equipados, é medir o comprimento de onda dos LEDs por meio da difração da luz por ele emitida através de uma rede de difração cuja constante de rede é conhecida (e, portanto, a distância entre as fendas adjacentes, $d$ ) e utilizar a expressão de difração de uma rede, 


$$
d \operatorname{sen} \theta=\lambda
$$

de modo que, conhecida a distância $d$, e medido o ângulo de difração, $\theta$, se possa calcular o valor do comprimento de onda, $\lambda$, de cada LED. Esta atividade poderia ser realizada pelos próprios estudantes em um experimento anterior (um exemplo desse experimento, realizável com material de baixo custo, pode ser encontrado em MOURA et al., 2011).

\section{Conclusão}

O resultado obtido com esta proposta experimental pode ser considerado como satisfatório, visto que o valor da constante de Planck registrado na literatura encontra-se dentro da margem de erro do obtido neste trabalho. A construção do circuito, além de ser possível com materiais de fácil aquisição, envolve conhecimentos simples que o aluno aprende no curso de eletromagnetismo, em etapas anteriores, e sua aplicação à física moderna é uma forma de mostrar que os conhecimentos adquiridos podem ser utilizados em diversas outras circunstâncias.

Os resultados obtidos podem ser melhorados com instrumentos de medida mais precisos, entretanto, devemos ter em mente que a principal fonte de erro desse método está no valor do comprimento de onda utilizado para os LEDs, pois, devido ao processo de fabricação, estes valores podem variar dentro de um certo intervalo.

Ainda, como sugestão complementar de prática, pode-se realizar uma atividade experimental anterior na qual seriam determinados os comprimentos de onda dos diversos LEDs, com o uso de uma rede de difração, e, após isso, efetuar o experimento aqui proposto (para aqueles laboratórios que possuem os materiais necessários para tanto). Alternativamente, esta atividade experimental pode ser usada com a finalidade de se determinar os comprimentos de onda de diversos LEDs (uma vez conhecido $h$ ) usando, para tanto, o nosso procedimento para a determinação da tensão de corte e, pela equação (3), do comprimento de onda de máxima emissão.

\section{Referências}

ALVES, E. G.; SILVA, A. F. Usando um LED como fonte de energia. Física na Escola, v. 9, n. $1,2008$.

AZEVEDO, H. L. et al. O Uso do experimento no ensino de Física: Tendências a partir do levantamento dos periódicos da área no Brasil. In: ENCONTRO NACIONAL DE PESQUISA EM EDUCAÇÃO EM CIÊNCIAS, 7, 2009, Florianópolis. Anais... Florianó-poilis: ABRAPEC, 2009.

BAÚ DA ELETRÔNICA. Disponível em:

$<$ http://baudaeletronica.blogspot.com.br/2008/03/tabela-de-leds.html>. Acessado em: 19 jun. 2015. 
CAVALCANTE, M. A.; HAAG, R. Corpo negro e determinação experimental da constante de Planck. Revista Brasileira de Ensino de Física, v. 27, n. 3, p. 343-348, 2005.

COLINGE, J. P.; COLINGE, C. A. Physics of Semiconductor Devices. Dordrecht : Kluwer Academic Publishers, 2000.

EISBERG R.; RESNICK, R. Física Quântica. 9. ed. S.L.: Campus, 1994.

FORÇA, A. C.; LABURÚ, L. C.; SILVA, O. H. M. Atividades experimentais no ensino de Física: Teoria e Práticas. In: ENCONTRO NACIONAL DE PESQUISA EM EDUCAÇÃO EM CIÊNCIAS, 8, 2011, Campinas, Anais... Campinas: ABRAPEC, 2011.

HALLIDAY, D.; RESNICK, R.; WALKER, J. Física 4. 5. ed. Rio de Janeiro: LCT, 2004. p. 104.

HEWITT, P. G. Física Conceitual. Porto Alegre: Bookman, 2011.

HORIGUTI, A. M.; OLIVEIRA, I. Determinação da constante de Planck através de dispositivo emissor de luz (LED). Sinergia, v. 9, n. 1, p. 43-48, 2008.

INDELICATO, V. et al. Analysis of LED data for the measurement of Planck's constant in the undergraduate laboratory. European Journal of Physics, v. 34, p. 819-830, 2013.

KRAFTMAKHER, Y. Experiments with light-emitting diodes. American Journal of Physics, v. 74, n. 8, 2011.

MOHR, P. J.; TAYLOR, B. N.; NEWELL, D. B. CODATA Recommended values of the fundamental physical constants: 2010. Review of Modern Physics, v. 84, 2012.

MOREHOUSE, R. Answer to Question \#53. Measuring Planck's constant by means of an LED. American Journal of Physics, v. 66, n. 1, p. 12, 1998.

MOREIRA, A. C. S.; PENIDO, M. C. M. Sobre as propostas de utilização das atividades experimentais no ensino de Física. In: ENCONTRO NACIONAL DE PESQUISA EM EDUCAÇÃO EM CIÊNCIAS, 7, 2009, Florianópolis. Anais... Florianópoilis: ABRAPEC, 2009.

MOURA, S. L. Constante de Planck: Uma nova visão para o Ensino Médio. Química Nova na Escola, v. 33, 2011.

NUSSENZVEIG, H. M. Curso de Física Básica. 7. ed. São Paulo: Blucher, 2009. v. 3.

RAYCHAUDHURI B. On the determination of the emission wavelength of an infrared LED with common laboratory instruments, European Journal of Physics, v. 32, n. 4, p. 935, 2011. REZENDE, S. M. Materiais e Dispositivos Eletrônicos. SP: Livraria da Física, 2004. 
TAVOlaro, C. R. C; CAVAlCANTE, M. A. Física Moderna Experimental. SP : Manole Ltda, 2003.

THURMAN, Z. Determining Planck's Constant Using LEDs. Disponível em: <http://works.bepress.com/zechariah_thurman/2/>. Acesso em: 19 jun. 2015.

ZHOU, T.; CLONINGER, T. Computer-based experiment for determining Planck's constant using LEDs. The Physics Teacher, v. 46, p. 413-415, 2008. 